\title{
D/H-ratio: Observations with ORFEUS II
}

\author{
M. Gölz ${ }^{1}$, N. Kappelmann ${ }^{1}$, I. Appenzeller ${ }^{2}$, J. Barnstedt ${ }^{1}$, A. Fromm ${ }^{1}$, \\ M. Grewing ${ }^{3}$, W. Gringel ${ }^{1}$, C. Haas ${ }^{1}$, W. Hopfensitz ${ }^{1}$, G. Krämer ${ }^{1}$,

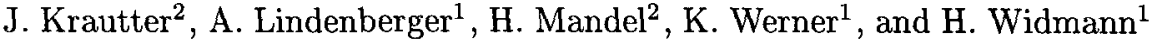 \\ 1 Institut für Astronomie und Astrophysik, Abt. Astronomie, \\ Waldhäuserstraße 64, D-72076 Tübingen, Germany \\ 2 Landessternwarte Heidelberg, Königsstuhl, D-69117 Heidelberg, Germany \\ ${ }^{3}$ Institut de Radio Astronomie Millimétrique (IRAM), 300 Rue de la Piscine, \\ F-38406 Saint Marint d'Hères, France
}

\begin{abstract}
During the second flight of the ORFEUS-SPAS satellite (Nov./Dec. 96) high resolution $\left(\frac{\lambda}{\Delta \lambda}=10,000\right)$ Echelle-spectra of $\mathrm{BD}+28^{\circ} 4211$ in the wavelength regime $912-1400 \AA$ have been taken. Deuterium can be clearly identified in the ORFEUS II Echelle-spectra of this star. For the first time it was possible to take spectra of faint, not redshifted objects in the far ultraviolet with a sufficient spectral resolution to study the deuterium column density in the whole spectral range of the Lyman-series down to the Lyman-limit. We obtained a value of $\log \left(\mathrm{N}_{\mathrm{D}}\right)=14.7( \pm 0.3)$ towards $\mathrm{BD}+28^{\circ} 4211$. The hydrogen column density has been determined using ORFEUS Echelle- and IUE-spectra of $\mathrm{Ly}-\alpha\left(\log \left(\mathrm{N}_{\mathrm{H}}\right)=\right.$ $19.8( \pm 0.2)$ ). Thus a value of $8 \times 10^{-6}$ can be obtained for the D/H-ratio on the line-of-sight towards $\mathrm{BD}+28^{\circ} 4211$.
\end{abstract}

\section{Introduction}

The interstellar abundance of deuterium is an indicator for the density of baryonic matter in the universe. McCullough (1992) has derived a unique interstellar $\mathrm{D} / \mathrm{H}$ value for the local ISM of $1.5( \pm 0.2) \times 10^{-5}$ by reevaluating Lyman absorption-line measurements made by the Copernicus and the IUE satellite. The interstellar $\mathrm{D} / \mathrm{H}$ ratio can be determined from the relative strength of the deuterium and hydrogen Lyman absorption lines, which are separated by $82 \mathrm{~km} \mathrm{~s}^{-1}$. The ORFEUS Echelle-spectrograph is the first instrument, which can access the whole Lyman-series down to the Lyman-limit with a sufficient spectral resolution to resolve the deuterium and hydrogen lines. Up to now only very few investigations of the deuterium column density have been made in the southern Milky Way in the vicinity of $\mathrm{BD}+28^{\circ} 4211$, a non-variable late-type object.

\section{Data}

The hot subdwarf $\mathrm{BD}+28^{\circ} 4211\left(\mathrm{~T}_{\text {eff }}=82,000 \mathrm{~K}\right.$ and $\log \mathrm{g}=6.2$, Napiwotzki 1993 ) is located at a distance of about $100 \mathrm{pc}$ (Perryman, priv.com.). Due 
to the preliminary data reduction we used only one out of several spectra of $\mathrm{BD}+28^{\circ} 4211$ taken during the ORFEUS II-mission from Nov. 19 to Dec. 04, 1996 using the ORFEUS Echelle-spectrograph in the wavelengthregime $\lambda \lambda=912-1400 \AA$ with a spectral resolution of $\frac{\lambda}{\Delta \lambda}=10,000$ (Krämer et al. 1990).

The spectrum of this hydrogen-rich subdwarf is dominated by stellar and interstellar Lyman lines of atomic hydrogen. The strongest line (Ly- $\alpha$ ) has been used for the determination of the hydrogen column density. The interstellar deuterium lines Ly- $\beta$ through Ly- $\eta(\lambda \lambda=1025.44-925.97 \AA)$ are clearly identified in the spectrum. The Ly- $\alpha$ deuterium-line $(\lambda=1215.34 \AA)$ is not visible due to strong geocoronal Ly- $\alpha$ emission (Fig. 1 (b)). We have used the deuterium lines Ly- $\beta$ through Ly- $\lambda$ for the determination of $\log \left(\mathrm{N}_{\mathrm{D}}\right)$.

The N I-triplet at $\lambda \approx 1134.5 \AA$, which is fully resolved in the ORFEUS II Echelle-spectrum, has been used to check for clouds with different velocities in the ISM on the line-of-sight (Fig. 1 (a)). The profile of the N I-triplet indicates that there are no different cloud components. Several absorption lines of NI in the ORFEUS Echelle-spectrum have been analysed to estimate the turbulence velocity within the ISM along the line-of-sight towards $\mathrm{BD}+28^{\circ} 4211$.

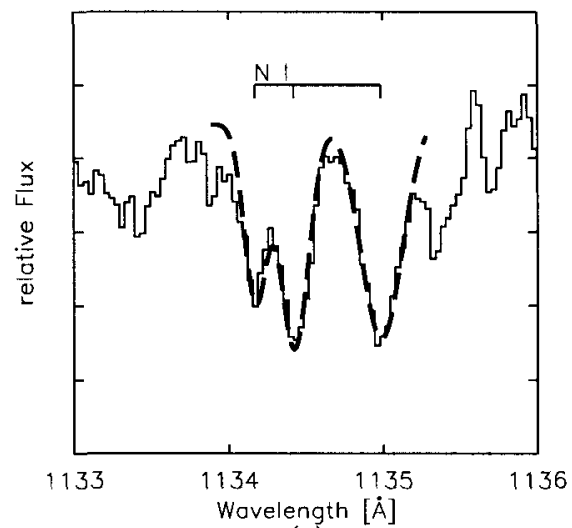

(a)

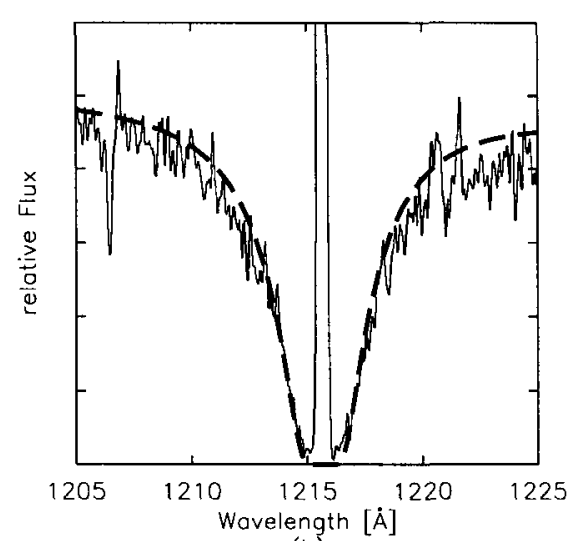

(b)

Fig. 1. (a) The N I-triplet at $\lambda \approx 1134.5 \AA$ in the ORFEUS II spectrum and a fit of the three components is shown (dashed curve). (b) The Ly- $\alpha$ line of hydrogen in the ORFEUS II spectrum of $\mathrm{BD}+28^{\circ} 4211$ and the theoretical line profile calculated for the value $\log \left(\mathrm{N}_{\mathrm{H}}\right)=19.8$ is presented (dashed curve). In the center of the Ly- $\alpha$ absorption the strong geocoronal emission of $\mathrm{Ly}-\alpha$ is visible. 


\section{Analysis and Results}

\subsection{Derivation of $\log \left(\mathrm{N}_{\mathrm{H}}\right)$}

In order to derive the hydrogen column density in the line-of-sight towards $\mathrm{BD}+28^{\circ} 4211$, both the Ly- $\alpha$ line of hydrogen and molecular hydrogen transitions have been analysed. The spectrum shows some evidence for a small amount of $\mathrm{H}_{2}$, which seems to be neglectable for the further analysis. The ORFEUS II Echelle-spectrum of $\mathrm{BD}+28^{\circ} 4211$, as well as a high resolution $I U E$-spectrum, have been analysed to determine the atomic hydrogen column density. In order to separate the stellar and the interstellar component, recent NLTE model-spectra with $\mathrm{T}_{\text {eff }}=80,000 \mathrm{~K}$ and $\log \mathrm{g}=6.25$ with an atmospheric composition of $n_{\mathrm{He}} / \mathrm{n}_{\mathrm{H}}=0.1$ have been used (Napiwotzki priv.com.). Natural damping-profiles with different interstellar column-densities have been applied to the stellar absorption lines and the resulting profiles have been compared with the observed ones (Fig. 1(b)). We obtained a value of $\log \left(\mathrm{N}_{\mathrm{H}}\right)=19.8( \pm 0.2)$ for the interstellar hydrogen column density.

\subsection{Derivation of $\log \left(\mathrm{N}_{\mathrm{D}}\right)$}

We analysed thirteen interstellar absorption lines of neutral nitrogen with the standard curve of growth technique. A turbulence velocity of $b=6 \mathrm{~km} \mathrm{~s}^{-1}$ in the ISM fits the data quite well. Based on this result we determined the theoretical equivalent widths of deuterium Ly- $\beta$ through Ly- $\lambda$ for different hydrogen column-densities using a standard curve of growth. For each absorption we fitted the nearby hydrogen-line together with a fixed set of parameters of the deuterium absorption to the observed profiles. The continuum has been corrected for stellar contribution using a recent atmospheric model of $\mathrm{BD}+28^{\circ} 4211$ (Napiwotzki, priv.com.).

The investigations showed, that the weak transitions of deuterium Ly- $\epsilon$ $(\lambda=937.5 \AA)$ through $\operatorname{Ly}-\lambda(\lambda=917.9 \AA)$ are most suitable for the estimation of $\log \left(\mathrm{N}_{\mathrm{D}}\right)$. Those lines are weak enough, that the line-profiles depend only on the column density and are independent from the turbulence velocity.

The strongest observable lines $(\mathrm{Ly}-\beta-\mathrm{Ly}-\delta)$ are dominated by the strong hydrogen absorption, which depletes the continuum, therefore the S/N- ratio of the deuterium absorption is reduced. Additionally those features are more sensitive to the background subtraction, which is only preliminary at this point. Furthermore, the equivalent widths of those strong deuterium lines depend already significantly on the turbulence velocity. Therefore we mainly stressed upon Ly- $\epsilon$ through $\mathrm{Ly}-\lambda$ for the determination of $\log \left(\mathrm{N}_{\mathrm{D}}\right)$. No further effort has been taken to derive a more precise value of $b$. A value of $\log \left(\mathrm{N}_{\mathrm{D}}\right)=14.7( \pm 0.3)$ has been determined from the analysed data. Although there is some evidence for $\mathrm{H}_{2}$ in the spectrum, no absorption of HD has been found. The $\mathrm{N}_{\mathrm{D}} / \mathrm{N}_{\mathrm{H}}$-ratio on the line-of-sight towards $\mathrm{BD}+28^{\circ} 4211$ can be given as $\mathrm{N}_{\mathrm{D}} / \mathrm{N}_{\mathrm{H}}=8\left({ }_{-4}^{+7}\right) \times 10^{-6}$. McCullough (1992) derived the mean value for the local ISM as $\mathrm{N}_{\mathrm{D}} / \mathrm{N}_{\mathrm{H}}=1.5( \pm 0.2) \times 10^{-5}$, which is compatible with the value derived in this publication. 


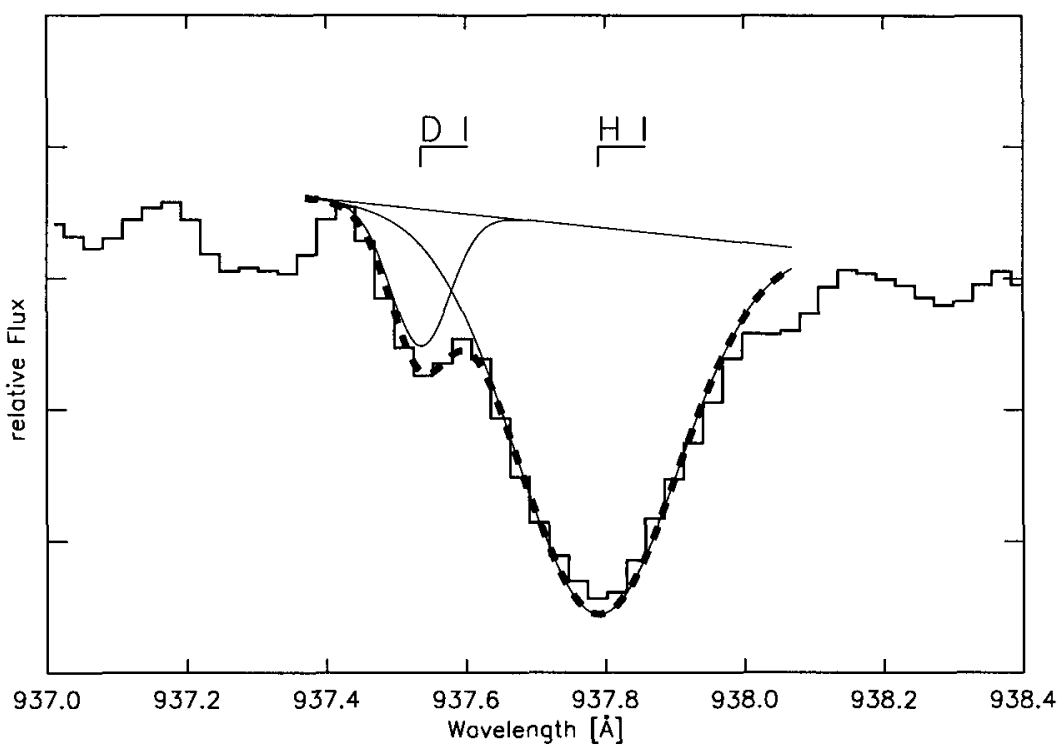

Fig. 2. The ORFEUSII spectrum (histogram mode) with a fit of deuterium and hydrogen Ly- $\epsilon$ (dashed curve) assuming a value of $\log \left(\mathrm{N}_{\mathrm{D}}\right)=14.7$. Both components and the centers of the absorption lines are indicated. The spectrum has been corrected for the stellar contribution.

Acknowledgements. We wish to thank Dr. Napiwotzki for calculating the atmosphere models for $\mathrm{BD}+28^{\circ} 4211$ and making them available to us and Dr. Perryman for supplying us the parallax of $\mathrm{BD}+28^{\circ} 4211$.

Special thanks to all American and German colleagues and collaborators, who helped to realize the successful ORFEUS-mission. The ORFEUS-program was supported by DARA grants WE3 OS 8501 and WE2-QV 9304 and NASA grant NAG5696.

\section{References}

Krämer, G. et al. (1990): Observatories in Earth Orbit and Beyond, ed. Y. Kondo (Kluwer Academic Publishers), 177

McCullough, P.R. (1992): ApJ 390, 213

Napiwotzki (1993): Acta Astron. 43, 343 